\title{
Home hemodialysis during the COVID-19 epidemic: comment on the French experience from the viewpoint of a French home hemodialysis care network
}

\author{
Pierre-Antoine Michel ${ }^{1} \cdot$ Giorgina Barbara Piccoli $^{2,3} \cdot$ Cécile Couchoud $^{4} \cdot$ Hafedh Fessi $^{1}$
}

Published online: 11 November 2020

(c) Italian Society of Nephrology 2020

Keywords SARS-CoV-2 $\cdot$ Home hemodialysis $\cdot$ France $\cdot$ Pandemic $\cdot$ COVID-19

A recent paper in Kidney International on the French experience with COVID-19 in dialysis patients states that between March 16 and May 4, 2020, of the 1621 infected patients listed in the REIN registry, 344 patients died, a confirmation of the high death toll among dialysis patients during the COVID-19 epidemic [1].

In this nationwide report, the prevalence of patients with COVID-19 varied between regions from less than $1 \%$ to over $10 \%$. The incidence of the disease was higher in males, and in patients with diabetes and other comorbidities, while being on dialysis at home was associated with a lower rate of infection. In fact, during the SARS-CoV-2 pandemic, numerous studies showed that end-stage kidney disease (ESKD) is a major risk factor for developing severe COVID-19 [2, 3].

Acknowledging this grim prognosis has been a stimulus for reconsidering dialysis policy from a different point of view [1, 3, 4]. Home dialysis has been advocated as a safer option in times of epidemic and, among others, the French health care authorities strongly supported the development of home-based techniques in their documents which

Hafedh Fessi

hafedh.fessi@aphp.fr

Pierre-Antoine Michel

pierre-antoine.michel@aphp.fr

Cécile Couchoud

cecile.couchoud@biomedecine.fr

1 Department of Nephrology and Dialysis, Hôpital TENON, Assistance Publique - Hôpitaux de Paris, Paris, France

2 Service of Nephrology, Centre Hospitalier LE MANS, Le Mans, France

3 Department of Clinical and Biological Sciences, University of Torino, Turin, Italy

4 Agence de La Biomedecine, Paris, France appeared in response to the COVID-19 epidemic [5, 6]. Potential drawbacks were also underlined, as were patients' need to have frequent contacts with a dialysis center and to avoid being isolated.

Home hemodialysis is still a Cinderella in Europe: while daily hemodialysis is considered to be one of the best renal replacement treatments, combining higher survival and a good quality of life, its penetration is limited. In France this technique has undergone a relatively rapid diffusion in the last 5 years. According to the data from the French Biomedicine Agency, during the peak period of the epidemic, between March 1 and June 15, 2020, only 7 of the 423 patients on home hemodialysis in France were diagnosed with COVID-19; the incidence is similar to that observed in patients treated with peritoneal dialysis (1.8\%). Even considering the different distribution of home dialysis in France (Fig. 1), the recorded incidence is significantly lower than that observed in patients on in-center hemodialysis (1856 COVID out of 48,840 patients, $3.8 \%$; $p=0.02$ ) [7]. Interestingly, the virus diffusion was the highest in the regions with the highest prevalence of home hemodialysis, and the favorable effect was more evident in settings with high virus circulation. For instance, in the Ile de France region, one of the most affected by the pandemic, data from the Biomedicine Agency indicate that 930 out of 8,025 patients (11.5\%) on in-center hemodialysis were diagnosed with COVID-19, whereas only 4 out of 109 patients on home hemodialysis developed the disease (3.6\%) $(p=0.001)$. Presumably because of the lower age (mean 54 years \pm 14 years) and comorbidity burden, none of the patients on home hemodialysis died. Furthermore, while home dialysis encompasses 7\% of all French dialysis patients, data from the French Biomedicine Agency show that the proportion of home dialysis patients (whether on hemodialysis or on peritoneal dialysis) represented $2.6 \%$ of the total number of dialysis patients 


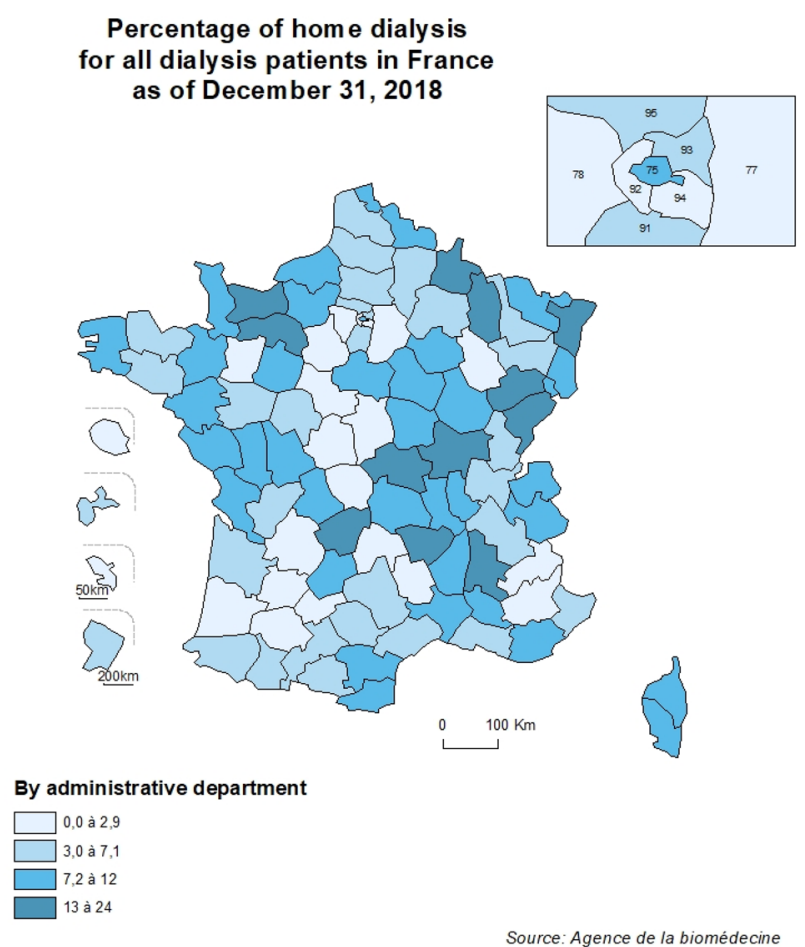

Fig. 1 Percentage of home dialysis for all dialysis patients in France as of December 31,2018. The numbers in the enlarged figure indicate administrative department

diagnosed with COVID-19 during the period March-October 2020 [64 out of 2437patients]; this proportion would appear to remain stable during the second epidemic wave.

The organization of a home hemodialysis network is not simple. In our network of care (based upon cooperation between the Tenon Hospital and the AURA Association) we are presently following 55 home hemodialysis patients, in a setting (Paris) that had a high virus circulation.

In our dialysis network, in normal conditions, outside of the epidemic, half of the patients who are trained for home hemodialysis are referred by our center and the other half by other centers. They are first evaluated by a nephrologist and a nursing team during a consultation in which our training policy is explained, and the advantages as well as the constraints of home treatment are set forth. The training period usually lasts eight weeks, during which the patients learn the management of dialysis and get accustomed to self-needling using the button-hole method. Training includes how to set up the generator, the management of alarms and how to adapt their dry weight. Nurses evaluate the patient's home and discuss how to solve possible issues (such as storage or waste management). The first dialysis session which is performed at home is organized in the presence of a nurse and a nephrologist. The disposables are sent once or twice a month to the patient's home by a non-profit association with experience in managing home hemodialysis in France (AURA for the Ile de France region), and waste retrieval is carried out weekly by a private company.

Each month one dialysis session is organized in the training center, thus providing an opportunity for blood tests to be done, as well as for clinical evaluation and discussion of any problems that may have occurred during the period. Our training unit is located on the same floor as the hemodialysis unit, but is separated from the main unit, with dedicated waiting rooms. The nursing team is entirely dedicated to supervising home hemodialysis.

During the COVID-19 epidemic, the dialysis network needed to be adapted, balancing the need for closely monitoring the fragile patients' clinical condition, with the need to minimize their exposure to the hospital [4]. Providing home assistance by a trained nurse, as is routinely done in peritoneal dialysis, was not proposed, as in France this option is not allowed for home hemodialysis. Training for home hemodialysis was performed on an intensified daily basis, balancing the risks of frequent hospital visits with the advantage of receiving treatment in a safer setting. The risks of social isolation were kept in mind, and a policy of distant monitoring was established on a case-by-case basis. We were able to train eight patients during the first phase of the epidemic and to secure them on home hemodialysis.

The delivery of consumables for hemodialysis was carried out respecting the barrier measures. Access to interventional radiology for the management of vascular access complications remained operational in our region during the period of the study. The creation of a new vascular access was however limited to emergencies.

We also chose to maintain the regular dialysis sessions and controls in the training unit, but we increased the intervals between visits from the usual monthly frequency to once every 6-8 weeks, adapting the control schedule to each patient's circumstances. Conversely, patients who had technical problems or experienced vascular access difficulties were immediately referred to the training center to try to promptly solve the problems. We considered that in such cases direct interaction played an important role in reassuring patients, and this advantage was balanced against their exposure to a hospital setting, with an increased risk of contamination. The longer intervals between visits were compensated by regular phone contacts, in order to strengthen monitoring, to reassure our patients and to remind them of the importance of respecting the barrier measures and employing personal protective equipment whenever they were outside their homes.

Of our 55 patients, 15 presented symptoms consistent with a viral infection. They all underwent a SARS-CoV-2 PCR. Two tested positive, 13 negative. Furthermore, globally 52 of our 55 patients later agreed to have a SARS-CoV-2 serology test performed, which was positive in the 2 patients 
who had previously tested positive to SARS-CoV-2 and negative in the remaining 50 patients.

Therefore, within the limits of a small series, and while waiting for additional data, we would like to confirm that our experience underlines the importance of developing an efficient home hemodialysis network, able to patients in a time of epidemic.

The catastrophic COVID-19 pandemic will presumably not be the last one in our globalized world. Home hemodialysis allows effective containment, and this effect is particularly high in endemic areas, potentially reducing mortality in dialysis patients. This consideration should further support the development of home hemodialysis, overcoming the many barriers to its development. Performing home hemodialysis, however, involves more than merely moving the dialysis machine from hospital to home. Developing a home hemodialysis network is a complex and demanding task, and in many European countries reimbursement fails to fully cover the costs, in particular if patients are closely monitored.

In France, for example, the average cost of a home dialysis session was estimated at $€ 221.78$, while the flat reimbursement rate for home hemodialysis is only $€ 225.15$. The lack of profit in home dialysis is a disincentive to its growth, despite its benefits for quality of life and, possibly, survival [8]. In addition to this problem, there are significant disparities in access to home dialysis among regions, mainly reflecting the involvement of each region's nephrology team. A lack of training of nephrologists and lack of information for patients are further important barriers to the development of home hemodialysis.

The present report, therefore, is a call for dedicated investment in home hemodialysis, and for an unbiased appraisal of its complexity, to implement the old, but always valuable concept of "home dialysis first" for patients who cannot receive a preemptive kidney transplantation [9].

Acknowledgements We would like to thank all the dialysis centers participating in the RDPLF and the REIN registries which, despite this difficult period, with a significant work overload, actively collected data and were available for additional information.

\section{Compliance with ethical standards}

Conflict of interest All authors declare that they have no conflicts of interest.
Ethics approval This article does not contain any studies with human participants performed by any authors.

\section{References}

1. Couchoud C, Bayer F, Ayav C, Béchade C, Brunet P, Chantrel F, Frimat L, Galland R, Hourmant M, Laurain E, Lobbedez T, Mercadal L, Moranne O (2020) in the name of the French REIN registry Low incidence of SARS-CoV-2, risk factors of mortality and the course of illness in the French national cohort of dialysis patients., Kidney Int. doi: https://doi.org/10.1016/j. kint.2020.07.042.

2. Quintaliani G, Reboldi G, Di Napoli A, Nordio M, Limido A, Aucella F, Messa P, Brunori G, Italian Society of Nephrology COVID-19 Research Group (2020) . Exposure to novel coronavirus in patients on renal replacement therapy during the exponential phase of COVID-19 pandemic: survey of the Italian Society of Nephrology. J Nephrol. 3:1-12. https://doi.org/10.1007/s4062 0-020-00794-1 (Online ahead of print)

3. Ikizler TA, Kliger AS (2020) Minimizing the risk of COVID-19 among patients on dialysis. Nat Rev Nephrol. 16(6):311 3.

4. Stern LD, Waikar S (2020) Time to expand access and utilization of home dialysis: lessons from the COVID-19 pandemic. Mayo Clin Proc 95(7):1323-1324. https://doi.org/10.1016/j.mayoc p.2020.04.038 (Epub 2020 Jun 2)

5. Agence de la BioMedecine. Situation de l'épidémie de Covid-19 chez les patients dialysés et greffés rénaux en France [Internet]. 2020. Disponible sur: https://www.agence-biomedecine.fr/R-E-IN-Reseau-Epidemiologique-et-Information-en-Nephrologie

6. Adaptation de l'offre de prise en charge des Patients atteints d'insuffisance rénale au stade de suppléance dans le contexte de l'épidémie covid-19 https://solidarites-sante.gouv.fr/IMG/pdf/ prise-en-charge-patients-irc_covid-19.pdf

7. Nouvier M, Verger C, Fouque D (2020) Situation of the Covid19 epidemic in patients on peritoneal dialysis on 2020/05/15 in France: RDPLF data-base. Bull Dial Domic. 3(2):73-81. https:// doi.org/10.25796/bdd.v3i2.55433

8. Agar JWM, Barraclough KA, Piccoli GB (2019) Home haemodialysis: how it began, where it went wrong, and what it may yet be. J Nephrol 32(3):331-333

9. Oreopoulos DG, Thodis E, Passadakis P, Vargemezis V (2009) Home dialysis as a first option: a new paradigm. Int Urol Nephrol 41(3):595-605

Publisher's Note Springer Nature remains neutral with regard to jurisdictional claims in published maps and institutional affiliations. 